\title{
THIN FINITE SIMPLE GROUPS
}

\author{
BY MICHAEL ASCHBACHER ${ }^{1}$
}

Communicated by Olga Taussky Todd, January 28, 1976

Let $G$ be a finite group. Define $e(G)$ to be the maximum of the $p$-ranks $m_{p}(M)$ as $M$ ranges over 2-local subgroups of $G$ and $p$ ranges over all odd primes. $G$ is thin if $e(G)=1$. The following classification is obtained:

THEOREM. Let $G$ be a thin finite simple group. Then $G$ is isomorphic to $L_{2}(q), L_{3}(p), p=1+2^{a} 3^{b}, U_{3}(p), p=-1+2^{a} 3^{b}, b=0$ or $1, p$ an odd prime, $S z\left(2^{n}\right), U_{3}\left(2^{n}\right), L_{3}(4),{ }^{3} D_{4}(2),{ }^{2} F_{4}(2)^{\prime}, M_{11}$, or $J_{1}$.

$G$ is of characteristic 2 type if $F^{*}(M)$ is a 2-group for each 2-local subgroup $M$ of $G$. It appears that in the near future the problem of classifying the finite simple groups will be reduced to the determination of groups of characteristic 2 type. Those familar with the $N$-group paper will recognize that the characteristic 2 type classification can be naturally subdivided into the cases $e(G)=1$, $e(G)=2$, and $e(G) \geqslant 3$. Hence the thin group classification may be regarded as one step in the program to classify the finite simple groups.

The proof is quite lengthy and will appear elsewhere.

DEPARTMENT OF MATHEMATICS, CALIFORNIA INSTITUTE OF TECHNOLOGY, PASADENA, CALIFORNIA 91125

AMS (MOS) subject classifications (1970). Primary $20 \mathrm{D} 05$.

${ }^{1}$ Partial support supplied by the Alfred P. Sloan Foundation and the National Science Foundation. 